DOI 10.18699/PlantGen2019-139

\title{
Bioinformatics analysis of the structures of CRISPR/Cas-systems in the genomes of phytopathogenic bacteria
}

\author{
Portnaia I.A. ${ }^{1 *}$, Borisenko A.Yu. ${ }^{1}$, Dzhioev Yu.P. ${ }^{2}$ \\ ${ }^{1}$ Irkutsk State Medical University, Irkutsk, Russia \\ ${ }^{2}$ Laboratory Institute of Biomedical Technologies, Irkutsk, Russia \\ *e-mail:portnaya.yana.1997@yandex.ru
}

The study of phytopathogenic bacteria is a topical issue in most discussion. Since that time, when microorganisms disrupt the carbohydrate and protein exchanges of a plant. The object of the study is full genome sequence of the Agrobacterium fabrum str.C58 chromosome circular, downloaded from the RefSeq database (NC_003062.2.). Pathogenic strains of Agrobacterium spp. carry at least one $\mathrm{Ti}$ or $\mathrm{Ri}$ - plasmid. To date, to avoid mass death of plants, targeted genetically modified approaches are being developed, for example, CRISPR/Cas. To find CRISPR/Cas-systems MacSyFinder were used. The research for the structural and functional characteristics of the Cas-genes was carried out using the HMMER and makeblastdb software. CRISPI, CRISPRFinder, CRISPRDetect were used to select CRISPR-arrays in the genome of the bacterium. To looking for phages through decoded spacer sequences was taken the BLASTn. The results of the bioinformatics study have showed the following: 1 CRISPR-array was found, having 3 spacer sections separated by repeat. Spacers are 18 b.p., repeat -5 b.p. The total length of the CRISPR-array was 510 b. p. with repeat (C-GGCGGCTGTTCGGCAGG). The obtained spacer sites were analyzed in the CRISPR-array for phage identification in such software: Phages, CRISPRTarget. It was possible to identify the belonging of the spacers to the phages of Mycobacterium Phage Shipwreck (13 repeats), Gordonia phage GMA2 (15 repeats). Also, using the BLASTn, we have found that Agrobacterium fabrum has full identity with Plantactinospora sp. BC1 chromosome, Streptomyces sp. 1H-SSA4 genome, Xanthomonas perforans 91-118 etc. These results help us to suggest that, in addition to virulent plasmids, the total repeat plays a role in pathogenicity trough the CRISPR-array. Thus, the bioinformatics analysis of the CRISPR/Cas-system of the phytopathogenic bacterium Agrobacterium fabrum str.C58 chromosome circular made it possible to find out which part of the DNA in the bacterium in order to change its pathogenic properties. 